\title{
Polarization-locked temporal vector solitons in a fiber laser: theory
}

\author{
J. M. Soto-Crespo \\ Instituto de Óptica, Consejo Superior de Investigaciones Cientificas, Serrano 121, 28006 Madrid, Spain
}

N. N. Akhmediev

Australian Photonics Cooperative Research Centre, Optical Sciences Centre, Australian National University, Canberra ACT 0200, Australia

B. C. Collings*

Electrical Engineering Department, Princeton University, Princeton, New Jersey 08544

\section{S. T. Cundiff}

JILA, University of Colorado and National Institute of Standards and Technology, Boulder, Colorado 80309-0440

\section{K. Bergman}

Department of Electrical Engineering, Princeton University, Princeton, New Jersey 08544

\section{W. H. Knox}

Bell Laboratories, Lucent Technologies, Holmdel, New Jersey 07733

Received May 3, 1999; revised manuscript received October 18, 1999

\begin{abstract}
Stable polarization-locked temporal vector solitons are found in a saturable absorber mode-locked fiber laser with weak cavity birefringence. The system is theoretically modeled with two coupled complex GinzburgLandau equations that include fiber birefringence, spectral filtering, saturable gain, and slow saturable absorption. The solutions to this system are similar to those for elliptically polarized vector solitons in a lossless birefringent fiber. Numerical simulations show qualitative agreement with experimental results. (c) 2000 Optical Society of America [S0740-3224(00)00603-2]

OCIS codes: $190.5530,260.1440,260.5210,140.3510,140.4050$.
\end{abstract}

\section{INTRODUCTION}

Although single-mode fiber supports two orthogonal polarization states, the vectorial nature of light is often ignored in the discussion and theoretical model of optical solitons, because a scalar treatment is typically sufficient for the conditions of many systems of interest. However, polarization effects have to be taken into account if the fiber is homogeneously birefringent. ${ }^{1,2}$ Nonlinear coupling between the two polarization modes results in several interesting and important phenomena for optical solitons that do not occur in the absence of any nonlinearity. Menyuk first described how this nonlinear coupling can allow the envelopes of two orthogonally polarized components of a soliton to propagate in a highly birefringent environment without suffering temporal walk-off. ${ }^{3-5}$ This nonlinear coupling permits optical solitons to be significantly robust to the pulse-broadening effects of polarization-mode dispersion. ${ }^{6,7}$ Furthermore, theoretical studies have predicted the existence in low birefringent fibers of pulses that can propagate in a linearly birefringent environment without experiencing changes to their polarization state. These pulses use the nonlinear coupling between the two polarization components to directly compensate and cancel their different phase velocities. $^{8,9}$ It has been shown that, in addition to "scalar" solitons with a single polarization component along the fast or slow axis, there are vector soliton solutions that are elliptically polarized and exist above a certain threshold energy. This branch of elliptically polarized soliton solutions bifurcates from the branch of solitons linearly polarized along the fast axis. We note that these solitons are single solitons distributed between the two axes, in contrast to vector solitons found in Refs. 6, 10, and 11 , which are, in effect, two soliton solutions. ${ }^{12}$ In this sense, elliptically polarized solitons are ground-state nonlinear modes of the birefringent fiber, although they require some threshold energy for their existence. The experimental investigation of these last novel states, although of great interest, has been difficult to achieve.

Passively mode-locked fiber lasers ${ }^{13-24}$ are unique sources of ultrashort optical pulses for telecommunications and other applications. The pulses that they gen- 
erate can typically be characterized as solitons independent of the passive mode-locking method. The vectorial nature of these pulses also becomes important if birefringent elements are included in the cavity. ${ }^{25}$ Moreover, the presence of both birefringence and gain-loss in the system can give rise to new phenomena.

In this paper we investigate the behavior of the state of polarization of pulses in a mode-locked fiber laser with linearly birefringent fiber. Earlier ${ }^{26}$ we found that such laser systems reveal a multiplicity of various phenomena related to the soliton generation with two polarization components. In contrast to Ref. 26, in which a set of various periodic and stationary solutions were found, here we are interested only in elliptically polarized solitons. The polarization-locked vector soliton in a laser system is formed as a result of the balance between nonlinearity, birefringence, and dispersion on the one hand and the balance of gain and loss on the other. We observe that the former balance essentially determines the soliton profile and the relation between its polarization components. The latter balance fixes the amplitude, and hence the energy, of the soliton, selecting one from the whole family of elliptically polarized solutions. The whole family of elliptically polarized solitons can be investigated by varying the cavity parameters. In our experiments the key variable parameter is the magnitude of the birefringence, which is determined by a sequence of three pieces of birefringent fiber with variable angles between their principal axes.

The evolution of the state of polarization of solitons in a weakly birefringent fiber has been studied experimentally by Barad and Silberberg. ${ }^{27}$ They showed experimentally that the total polarization state of a soliton evolves as a unit and that solitons polarized along the fast axis are unstable. Moreover, they investigated the evolution of the state of polarization of solitons in a homogeneous birefringent fiber and found that it describes periodic orbits on the Poincaré sphere in accordance with the approximation of an average profile. ${ }^{28}$ However, stationary elliptically polarized solitons were not observed. Experimental investigation of the family of these solitons has been difficult owing to the weak instability of the stationary elliptically polarized solitons. This difficulty can be overcome by placing the birefringent fiber within a laser cavity. The laser is a self-regulated system generating highenergy pulses whose characteristics are determined by the parameters of the cavity, including the birefringence. Hence the intracavity pulses can be seen as the nonlinear modes of the total system.

Polarization-locking effects were experimentally discovered by Cundiff et al. ${ }^{25}$ in a weakly birefringent fiber laser cavity. In particular, the two polarization components of the intracavity pulse lock in phase. These pulses have been named polarization-locked vector solitons (PLVS's). ${ }^{29,30}$ Within a certain range of cavity parameters, the laser generates PLVS's that are stationary elliptically polarized solitons. In a companion paper ${ }^{31}$ it is experimentally shown that for certain values of birefringence and pulse energy the two components of the vector solitons formed in a passively mode-locked laser propagate with a constant relative optical phase of $\pm \pi / 2$. These pulses are then experimentally characterized and compared with the theoretical PLVS solutions of lossless fiber. Other polarization effects in slightly different configurations have also been reported. ${ }^{32}$

Here we numerically compare the PLVS solutions of a lossless fiber system with the solutions to the complete model of the experimental fiber laser. We illustrate a high degree of agreement between the two solution sets. This confirms that the experimentally observed polarization-locked pulses are indeed PLVS's of the cavity fiber and are not driven by an effect intrinsic to the nonconservative laser system. ${ }^{31}$ The main difference between the PLVS's in a lossless birefringent fiber and those in a laser cavity is their stability properties. In a lossless fiber, elliptically polarized solitons are weakly unstable owing to their interaction with background radiation. ${ }^{8,9}$ In the case of the laser, we choose the laser parameters such that the elliptically polarized solitons have the same properties as those for the lossless fiber, but, as will be shown, they become stable in a cavity. In our cavity model the instability that is due to radiation losses is included in the overall balance between gain and loss. Thus the growth of this instability is controlled by the mode-locking mechanism responsible for the stable generation of the pulse.

\section{MASTER EQUATION}

For analyzing the passively mode-locked laser, we use a set of two coupled complex Ginzburg-Landau equations for the two polarization components of the optical field. ${ }^{26}$ These equations include the terms responsible for the birefringence, amplification, and saturable losses. The laser is modeled as a distributed system, assuming that the pulse shape changes only slightly during each round trip. Explicitly, these equations governing the pulse evolution $\operatorname{are}^{26}$

$$
\begin{aligned}
& i \phi_{z}+\gamma \phi+\frac{D}{2} \phi_{t t}+|\phi|^{2} \phi+A|\psi|^{2} \phi+B \psi^{2} \phi^{*} \\
& =i\left[g\left(Q_{1}\right)-\delta_{l}-\delta_{s}\left(|\phi|^{2}\right)\right] \phi+i \beta \phi_{t t}, \\
& i \psi_{z}-\gamma \psi+\frac{D}{2} \psi_{t t}+|\psi|^{2} \psi+A|\phi|^{2} \psi+B \phi^{2} \psi^{*} \\
& =i\left[g\left(Q_{2}\right)-\delta_{l}-\delta_{s}\left(|\psi|^{2}\right)\right] \psi+i \beta \psi_{t t},
\end{aligned}
$$

where $z$ is normalized to the cavity length $Z_{0} ; t$ is the normalized retarded time $t=\left[T-\left(z /\left(V_{g} Z_{0}\right)\right] / T_{0}\right.$, where $T_{0}=\left(Z_{0}\left|\beta_{2}\right|\right)^{1 / 2} ; \quad \beta_{2}$ is the intracavity group-velocity dispersion; $\psi$ and $\phi$ are the normalized envelopes of the two optical field components; $\gamma$ is the half-difference between the propagation constants of the two components of the field; $D=\beta_{2} /\left|\beta_{2}\right|$ (= $=-1$ in our case); $A$ is the crossphase modulation coefficient; $B$ is the coefficient of the energy-exchange term (four-wave mixing); $\beta$ represents the spectral filtering or bandwidth-limited gain $(\beta>0)$ (for very short pulses, a better approximation is the Lorentzian gain line, ${ }^{33,34}$ but we consider the spectral width to be well inside a parabolic gain profile); $g\left(Q_{i}\right)$ is the gain in the cavity, which depends on the energy; $Q_{1}$ $=\int_{-\infty}^{\infty}\left(|\phi|^{2}\right) \mathrm{d} t ; \quad Q_{2}=\int_{-\infty}^{\infty}\left(|\psi|^{2}\right) \mathrm{d} t ; \quad \delta_{l}$ is the linear loss 
term; and $\delta_{s}$ represents the loss that is due to the semiconductor saturable absorber.

The gain term, $g\left(Q_{i}\right)$, in Eqs. (1) describes an active medium with a recovery time much longer than the round-trip time of the cavity and therefore does not depend explicitly on $t$. It describes depletion of the gain medium and depends on the partial pulse energy

$$
g\left(Q_{i}\right)=\frac{g_{0}}{1+Q_{i} / E_{L}},
$$

where $g_{0}$ is the small signal gain and $E_{L}$ is the saturation energy. The absorption of the saturable absorber is described by the following rate equation ${ }^{22}$ :

$$
\frac{\partial \delta_{s}}{\partial t}=-\frac{\delta_{s}-\delta_{0}}{T_{1}}-\frac{|\Upsilon|^{2}}{E_{A}} \delta_{s},
$$

where $T_{1}$ is the recovery time of the saturable absorber, $\delta_{0}$ is the loss introduced by the absorber in the absence of pulses, $Y$ refers to $\psi$ or $\phi$, and $E_{A}$ is the saturation energy of the absorber.

The solution of Eq. (2) can be written in a general form $^{35}$ and can be substituted directly into Eqs. (1) to take into account the slow effects of the saturable absorber. Owing to the symmetry of the propagation equation, if $(\phi, \psi)$ is a solution, then $(\phi,-\psi)$ is also a solution. We note the special choice of the gain-loss terms in the righthand side of Eqs. (1). Namely, those terms that are responsible for the gain or loss of one component depend only on the same component. This makes our analytical model slightly anisotropic. As shown below, this anisotropy can be removed in the numerical simulations.

\section{NUMERICAL RESULTS}

The numerical PLVS solutions are obtained as follows. ${ }^{36}$ We start with $\gamma=0$ and find the numerical soliton solution for that case. Then using this solution as an initial condition, we increase the birefringence and allow the polarization state (see Ref. 26) to converge to another fixed stable state, provided that it exists. Above a certain value of the birefringence, the polarization ceases to lock and evolves according to the birefringence.

For $\gamma=0$, a unique, stable, symmetrically degenerate solution exists with equal amplitude polarization components and $\pm \pi / 2 \mathrm{rad}$ out of phase. The energy, $Q=Q_{1}$ $+Q_{2}$, of this solution depends on the gain-loss parameters. Different values for this set of gain-loss parameters can produce the same solution. Figure 1 shows (a) $Q$ versus birefringence and (b) normalized energy difference, $\left(\int|\phi|^{2} \mathrm{~d} t-\int|\psi|^{2} \mathrm{~d} t\right) /\left(\int|\phi|^{2} \mathrm{~d} t+\int|\psi|^{2} \mathrm{~d} t\right)$, versus birefringence, for four values of the gain-loss parameters:

$$
\begin{aligned}
\text { (1) } E_{L} & =0.5, \quad \delta_{0}=0.12, \quad T_{1}=0.5, \quad E_{A}=0.4, \\
\beta & =0.02, \quad \text { and } g_{0}=0.327, \\
\text { (2) } E_{L} & =0.4, \quad \delta_{0}=0.07, \\
T_{1} & =0.5, \quad E_{A}=0.04, \\
\beta & =0.04, \quad \text { and } g_{0}=0.145,
\end{aligned}
$$
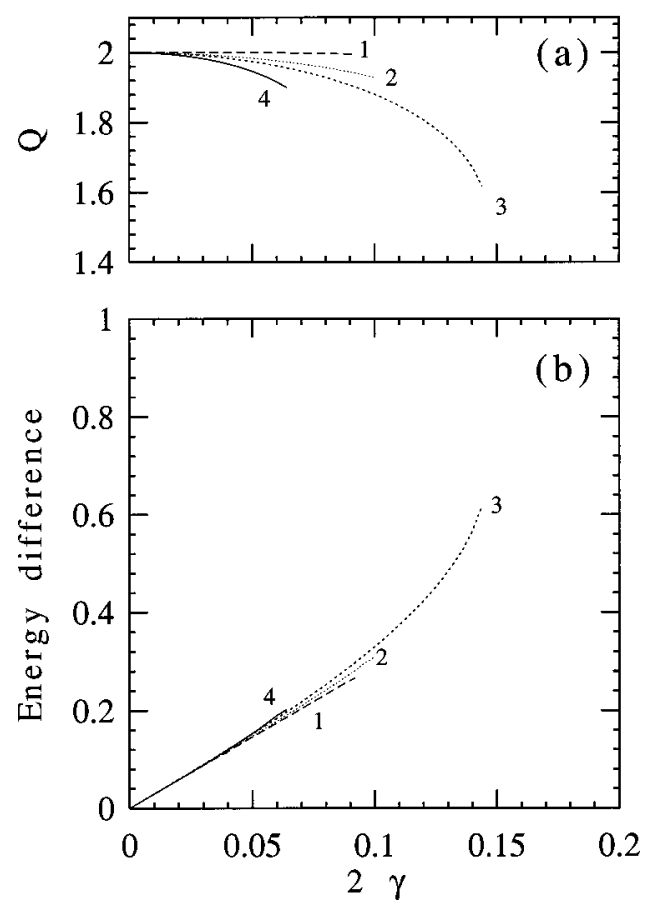

Fig. 1. (a) Total pulse energy versus birefringence for the stable elliptically polarized solitons. (b) Normalized energy difference between components versus birefringence. The curves end where the elliptically polarized solitons become unstable. The four curves correspond to four different sets of gain-loss parameters. See text.

(3)

$$
\begin{array}{rlrl}
\text { (3) } E_{L} & =0.5, & \delta_{0}=0.08, \\
T_{1} & =0.3, \quad E_{A}=0.018, \\
\beta & =0.1, \quad \text { and } g_{0}=0.137, \\
\text { (4) } E_{L} & =0.5, \quad \delta_{0}=0.08, \\
T_{1} & =0.5, \quad E_{A}=0.04, \\
\beta & =0.02, \quad & \text { and } g_{0}=0.115 .
\end{array}
$$

The remainder of the parameters, common for all four cases, are $\delta_{l}=0.01, A=2 / 3, B=1 / 3$, and $D=-1$. These four sets of parameters are chosen such that they produce the same stable, circularly polarized pulselike solution with $Q=2$ for $\gamma=0$.

As the birefringence $(\gamma)$ is increased, the two components of the solution converge to the solution corresponding to that $\gamma$. The difference between the components increases by increasing $\gamma$. The gain-loss terms influence them differently, depending on the precise values of the parameters. The results are shown in Fig. 1. It can be seen that the energy, $Q$, of the elliptically polarized soliton solutions obtained from numerical simulations generally decreases with $\gamma$ and the functions $Q(\gamma)$ are different for the four sets of parameters even if for each parameter set $Q(0)=2$. The energy difference between the components also changes with $\gamma$ but differently in the four cases. However, these curves are relatively close to each other. The main difference between the curves is that each has an upper limit that depends strongly on the values of parameters. This upper-limit difference occurs because the elliptically polarized soliton solution loses its 
stability at different values of $\gamma$ for each case. Thus the gain-loss terms play a crucial role in the stability of these solutions. $Q$-switching instabilities such as those reported in Ref. 37 were not observed in any of our simulations.

The set of Eqs. (1) contains a gain-loss anisotropy that is absent in the experiment. Even in the absence of birefringence $(\gamma=0)$, the model assumes that the medium has two equivalent, but distinct, directions: fast and slow axes. The problem is that the nonconservative terms in Eqs. (1) for each component depend only on that respective component. Linearly polarized light along any other direction would suffer different gain or loss from that polarized along either the fast or slow axis. An anisotropy may exist in the experiment owing to the polarization of the pump ${ }^{38}$ or polarization hole burning, but it should be rather weak. We assume that the gain and loss in the transverse plane in our experiment is isotropic.

The isotropy of the gain-loss terms can be introduced numerically into equations, that is, by evaluating the gain-loss terms uniformly along all possible azimuthal directions. We use a split-step Fourier method with the following modification. We rotate the axes for a certain angle $\theta$ at each half-step of propagation when the gainloss terms are nonzero and rotate them back for an angle $-\theta$ before the next half-step with zero gain-loss terms in Eqs. (1). With this procedure, the axes of birefringence and the components are unchanged. We randomly choose for each step a $\theta$ over the range of 0 to $\pi$, with constant density of probability. This procedure is equivalent to the randomization of the anisotropy in the location of erbium atoms in a glass host matrix. The gain and loss are now locally anisotropic, but isotropic in average.

Figure 2 shows the numerical results for $Q$ and for the energy difference versus $\gamma$ obtained with this isotropic model for the same sets of parameters as those in Fig. 1.
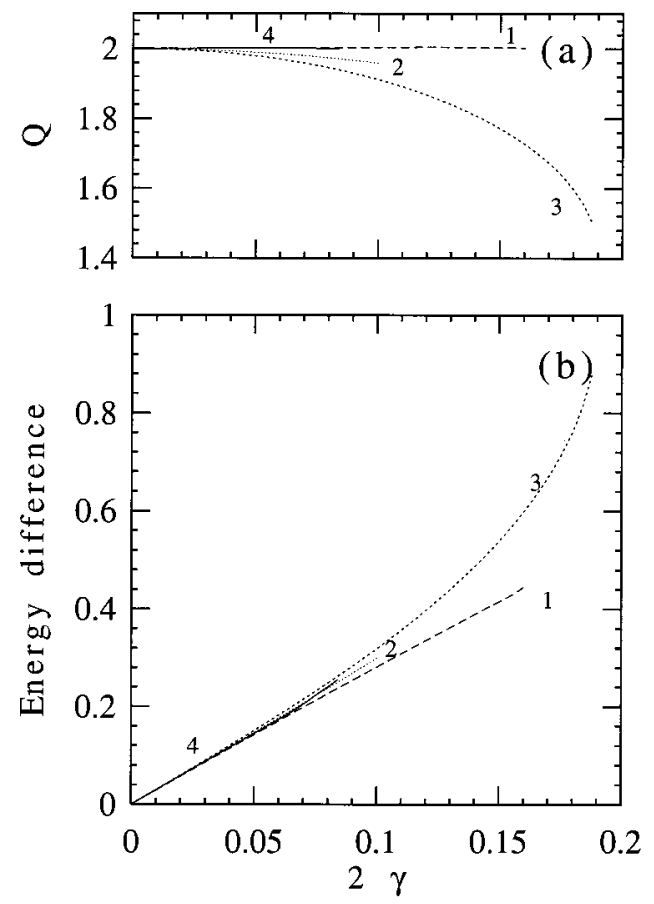

Fig. 2. Same as in Fig. 1 but with isotropic gain and loss.

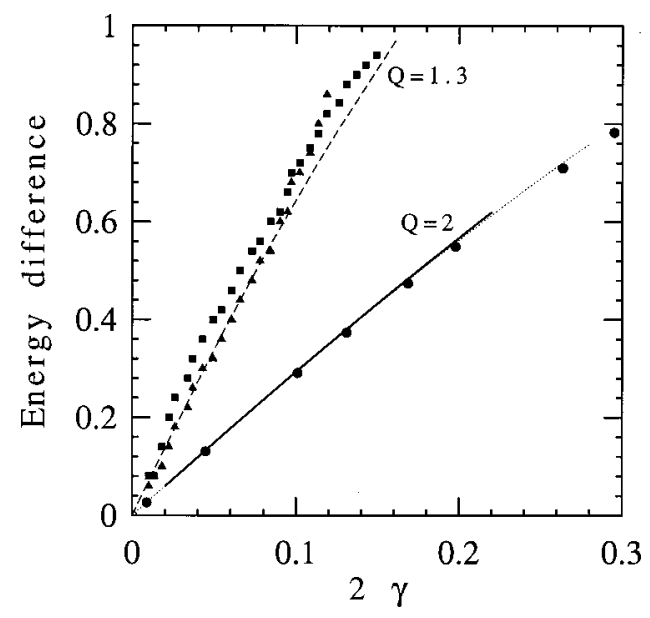

Fig. 3. Comparison between the energy difference versus birefringence for the solutions of the conservative case (solid circles) and our laser system with gain and loss by use of the anisotropic (continuous curve) and isotropic (dotted curve) models with the proper gain to keep $Q=2$. The dashed curve shows the same for $Q=1.3$ for the conservative solutions. The solid triangles and squares are the experimental data for both handednesses.

For the sake of comparison, the scales of the two plots coincide. The most prominent difference between the isotropic and anisotropic models is that in the isotropic model the curves are extended to larger birefringence. This indicates that adding gain-loss isotropy increases the range in which the elliptically polarized solitons are stable. This is expected, since the gain-loss terms can be considered a perturbation of the conservative terms of the equations. The main impact of the isotropy is on the growth rate of the nonlinear modes. The modes themselves should be primarily defined by the conservative parts of the model.

In our laser system the nonconservative terms are small and serve mainly to fix the soliton amplitude. The rest of the soliton parameters are determined by the conservative terms of nonlinearity, dispersion, and the birefringence. This suggests that the comparison with the conservative case ${ }^{8,9}$ should be done on the following basis. In the case of the above-cited Hamiltonian system (i.e., that described by the left-hand side of Eqs. 1 set to zero), the PLVS's comprise a one-free-parameter family of solutions. The parameter of the family is the propagation constant $q$. The energy $Q$, the energy difference, and any other pulse characteristics depend on this parameter. On the other hand, because of the scaling properties of this Hamiltonian system, if we fix the energy, then the energy difference will depend on $\gamma$. Let us recall that $Q / \sqrt{\gamma}$ depends exclusively on $q / \gamma$ [see Fig. 1(b) of Ref. 9]. In the experiment the pulse energy does not significantly depend on $\gamma$, although the remainder of the laser parameters are fixed, thus providing the basis for comparison with those solutions found in Refs. 8 and 9 .

In the simulations we fix the value of $Q$ by varying the small signal gain, $g_{0}$, while fixing the rest of parameters. The gain must be increased only slightly by increasing $\gamma$ in order to obtain a PLVS of constant energy. Figure 3 shows the normalized energy difference versus $\gamma$ for solutions with $Q=2$. The solid curve corresponds to the anisotropic case, and the dotted curve corresponds to the iso- 
tropic model described above. As we can see, the only difference between the anisotropic and isotropic cases is that, in the isotropic case, the PLVS remains stable over a larger interval of $\gamma$. The solid circles correspond to the conservative solutions obtained in Refs. 8 and 9. The agreement of these three curves is remarkable. The dashed curve is the corresponding curve for the conservative case with $Q=1.3$. Solid triangles and squares represent the experimental data for both handednesses. See the companion paper ${ }^{31}$ for more details.

The similarities among the solutions of Eqs. (1) and the conservative ones are not only in the energy difference between components but also in the rest of the pulse characteristics. Figure 4(a) shows the intensity profiles of the elliptically polarized soliton for $Q=2$ and $\gamma=0.1$ for the nonconservative laser system (with use of either the isotropic or anisotropic model) (dotted curve) and the conservative case (dashed curve). Figure 4(b) shows the phase profiles of both components for the nonconservative case. For the conservative case, the phase profiles are constant with time. The variation of their phase profiles is very minor, which indicates that the nonconservative effects select one specific solution from the whole family of the conservative case and only slightly modifies its form. In addition, owing to the scaling properties of the propagation equations for the conservative case, any other curve representing energy difference versus birefringence for other values of $Q$ can be obtained from that of Fig. 3.

To compare the theoretical-numerical results with the experimental results, we must relate the nondimensional units to the real ones. For this purpose, we take ${ }^{39} n_{2}$ $=2.3610^{-20} \mathrm{~m}^{2} / \mathrm{W}$ and consider the effective area $A_{\text {eff }}$ to be $80 \mu \mathrm{m}^{2}$. For the experiment the mean value of the dispersion is $\beta_{2}=17 \mathrm{ps}^{2} / \mathrm{km}$ and the round-trip cavity length, $Z_{0}$, that we take as the length unit, is $\approx 4.2 \mathrm{~m}$. Hence the time unit is

$$
t_{0}=\sqrt{\left(z_{0}\left|\beta_{2}\right|\right)} \approx 340 \mathrm{fs} .
$$

The real energy, $E$, is related to $Q$ in the following way:

$$
E=\frac{Q t_{0}}{K},
$$

where

$$
K=\frac{2 \pi n_{2} t_{0}^{2}}{\lambda A_{\mathrm{eff}}\left|\beta_{2}\right|}=\frac{2 \pi n_{2} Z_{0}}{\lambda A_{\mathrm{eff}}}=0.005 \mathrm{~W}^{-1} .
$$

Therefore

$$
E=67.6 Q \mathrm{pJ}
$$

i.e., multiplying $Q$ by 67.6 transforms it to picojoules.

The total spectrum, defined as $|\widetilde{\phi}|^{2}+|\widetilde{\psi}|^{2}$, where the tilde indicates the Fourier transform, of the PLVS presented in Fig. 4 is shown in Fig. 5 by a dotted curve. The dashed curve in Fig. 5 represents the square of the function $2.06 \operatorname{sech}(2 \omega)$, which fits quite well with the numerically found spectrum. If the time unit $t_{0}$ is taken to be $340 \mathrm{fs}$, then the frequency unit will be $10^{12} / 0.680 \pi$ $=468 \mathrm{GHz}$. Figure 6 shows the spectral full width at half-maximum (FWHM) of both components versus birefringence. At higher $\gamma$ (see Fig. 3), the intensity of the fast component becomes greater relative to the slow one, narrower in time and wider in spectrum. The dependence of the slow component versus $\gamma$ is the opposite. The solid circles represent the FWHM of the conservative solutions. Again, the agreement is quite remarkable.

We have also studied the region of parameters in which the stable PLVS exists, using the isotropic model for gain and loss. As can be seen from Fig. 2, the region depends strongly on the gain-loss parameters. Figure 7 shows that region for some specific set of parameters, namely, those labeled (3) in Figs. 1 and 2. The value of $Q$ in this case is the energy of the solution at $\gamma=0$. It changes as $\gamma$ increases. Although the exact boundaries of the stability region depend on the precise values of the gain-loss parameters, the general pattern for other sets of param-
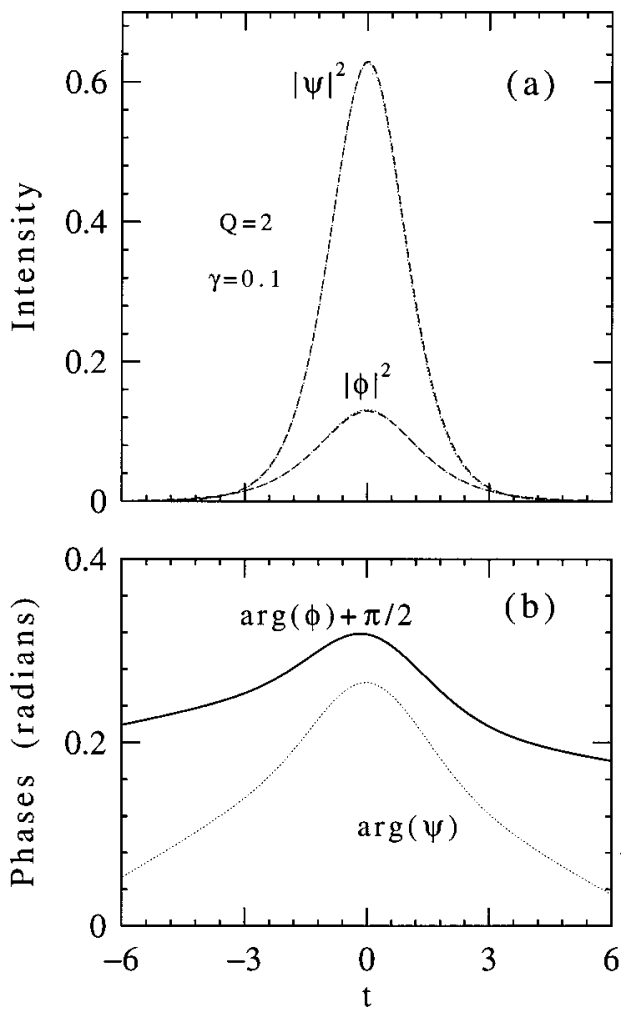

Fig. 4. (a) Intensity and (b) phase profiles of each component of the elliptically polarized solitons. (a) The solution of Eqs. (1) (dashed curve) is compared with that from the conservative case (dotted curve), which are almost indistinguishable.

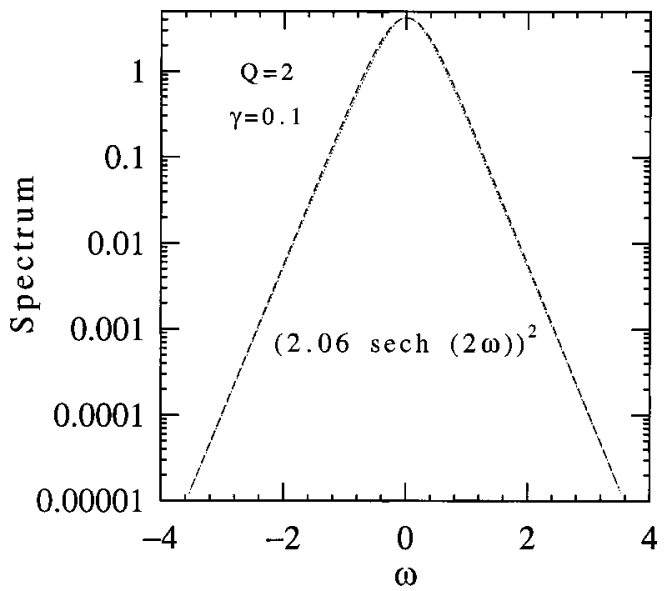

Fig. 5. Total spectrum of the solution shown in Fig. 4 . 


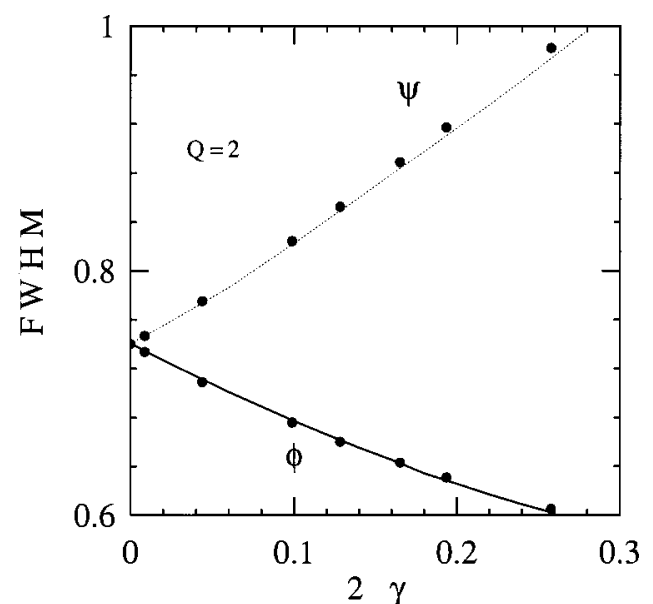

Fig. 6. The FWHM of the spectra of each component versus birefringence $\gamma$. Circles are calculated for elliptically polarized solitons in lossless fiber.

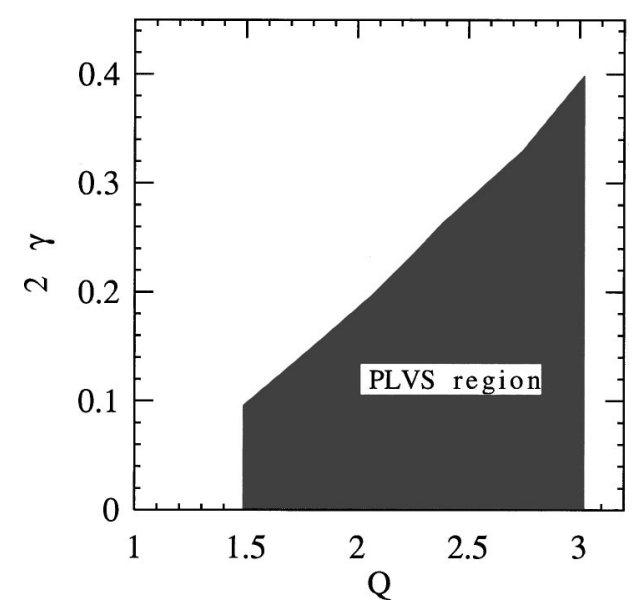

Fig. 7. The region of existence (shaded) of elliptically polarized soliton.

eters is similar to that shown in Fig. 7. The PLVS solution with higher-energy $Q$ exists in a larger range of $\gamma$ values. Exactly the same thing happens in the experiments (see Fig. 11 of Ref. 32). On the other hand, if the energy $Q$ is above a certain threshold, the pulse breaks up and creates several pulses inside the cavity.

\section{SUMMARY}

We have modeled a passively mode-locked laser with a saturable absorber and an intracavity birefringent fiber based on two coupled modified complex GinzburgLandau equations. We have shown that for the set of parameters with relatively weak gain-loss terms the PLVS's can be generated. These PLVS's have remarkably similar properties as the elliptically polarized solitons obtained for the conservative case except for their stability. Namely, PLVS's are stable in a range of values of the birefringence. The stability limit mainly depends on the soliton energy and gain-loss parameters. Experimental measurements confirm the dependency of soliton parameters on the value of birefringence in the cavity.

\section{ACKNOWLEDGMENTS}

The work of J. Soto-Crespo was supported by the Dirección General de Enseñanza Superior under contract PB96-0819. K. Bergman and B. Collings acknowledge support from the Office of Naval Research grant N0001496-0773 and the National Science Foundation grant ECS9502491.

*Present address, Bell Laboratories, Lucent Technologies, Holmdel, New Jersey 07733.

\section{REFERENCES}

1. C. R. Menyuk, "Pulse propagation in an elliptically birefringent Kerr medium," IEEE J. Quantum Electron. QE-25, 2674-2682 (1989)

2. K. J. Blow, N. J. Doran, and D. Wood, "Polarization instabilities for solitons in birefringent fibers," Opt. Lett. 12, 202-204 (1987).

3. C. R. Menyuk, "Nonlinear pulse-propagation in birefringent optical fiber," IEEE J. Quantum Electron. 23, 174-176 (1987).

4. C. R. Menyuk, "Stability of soliton in birefringent optical fibers. I. Equal propagation amplitudes," Opt. Lett. 12, 614-616 (1987).

5. C. R. Menyuk, "Stability of soliton in birefringent optical fibers. 2. Arbitrary amplitudes," J. Opt. Soc. Am. B 5, 392-402 (1988); 12, 614-616 (1988).

6. D. N. Christodoulides and R. I. Joseph, "Vector solitons in birefringent nonlinear dispersive media," Opt. Lett. 13, 53-55 (1988)

7. S. G. Evangelides, L. F. Mollenauer, J. P. Gordon, and N. S. Bergano, "Polarization multiplexing with solitons," J. Lightwave Technol. 10, 28-35 (1992).

8. N. N. Akhmediev, A. V. Buryak, and J. M. Soto-Crespo, "Elliptically polarised solitons in birefringent optical fibers," Opt. Commun. 112, 278-282 (1994).

9. N. N. Akhmediev, A. V. Buryak, J. M. Soto-Crespo, and D. R. Andersen, "Phase-locked stationary soliton states in birefringent nonlinear optical fibers," J. Opt. Soc. Am. B 12, 434-439 (1995).

10. M. V. Tratnik and J. E. Sipe, "Bound solitary waves in a birefringent optical fiber," Phys. Rev. A 38, 2011-2017 (1988).

11. N. N. Akhmediev, V. M. Eleonskii, N. E. Kulagin, and L. P. Shil'nikov, "Steady-state pulses in a birefringent nonlinear optical fiber: soliton multiplication processes," Sov. Tech. Phys. Lett. 15, 587-588 (1989).

12. N. Akhmediev and A. Ankiewicz, Solitons, Nonlinear Pulses and Beams (Chapman \& Hall, London, 1997).

13. I. N. Duling, "All-fiber ring soliton laser mode locked with a nonlinear mirror," Opt. Lett. 16, 539-541 (1991).

14. C.-J. Chen, P. K. A. Wai, and C. R. Menyuk, "Stability of passively mode-locked fiber lasers with fast saturable absorption," Opt. Lett. 19, 198-200 (1994).

15. H. A. Haus, J. G. Fujimoto, and E. P. Ippen, "Structures for additive pulse mode locking," J. Opt. Soc. Am. B 8, 20682076 (1991).

16. D. U. Noske, N. Pandit, and J. R. Taylor, "Subpicosecond soliton pulse formation from self-mode-locked erbium fibre laser using intensity dependent polarisation rotation," Electron. Lett. 28, 2185-2186 (1992).

17. P. A. Bélanger, "Coupled-cavity mode locking: a nonlinear model," J. Opt. Soc. Am. B 8, 2077-2081 (1991).

18. H. A. Haus, E. P. Ippen, and K. Tamura, "Additive-pulse modelocking in fiber lasers," IEEE J. Quantum Electron. 30, 200-208 (1994).

19. V. J. Matsas, D. J. Richardson, T. P. Newson, and D. N Payne, "Characterization of a self-starting passively modelocked fiber ring laser that exploits nonlinear polarization evolution," Opt. Lett. 18, 358-360 (1993). 
20. M. Hofer, M. E. Fermann, F. Haberl, M. H. Ober, and A. J. Schmidt, "Mode locking with cross-phase and self-phase modulator," Opt. Lett. 16, 502-504 (1991).

21. L. E. Nelson, D. J. Jones, K. Tamura, H. A. Haus, and E. P. Ippen, "Ultrashort-pulse fiber ring lasers," Appl. Phys. B 65, 277-294 (1997).

22. F. X. Kärtner and U. Keller, "Stabilization of solitonlike pulses with a slow saturable absorber," Opt. Lett. 20, 16-18 (1995).

23. B. C. Collings, K. Bergman, S. T. Cundiff, S. Tsuda, N. Kutz, J. E. Cunningham, W. Y. Jan, and W. H. Knox, IEEE J. Sel. Top. Quantum Electron. 3, 1065-1075 (1997).

24. J. N. Kutz, B. C. Collings, K. Bergman, S. Tsuda, S. T. Cundiff, W. H. Knox, P. Holmes, and M. Weinstein, "Modelocking pulse dynamics in a fiber laser with a saturable Bragg reflector," J. Opt. Soc. Am. B 14, 2681-2690 (1997).

25. S. T. Cundiff, B. C. Collings, and W. H. Knox, "Polarization locking in an isotropic, modelocked soliton $\mathrm{Er} / \mathrm{Yb}$ fiber laser," Opt. Express 1, 12-20 (1997).

26. N. N. Akhmediev, J. M. Soto-Crespo, S. T. Cundiff, B. C. Collings, and W. H. Knox, "Phase locking and periodic evolution of solitons in passively mode-locked fiber lasers with a semiconductor saturable absorber," Opt. Lett. 23, 852 854 (1998).

27. Y. Barad and Y. Silberberg, "Polarization evolution and polarization instability of solitons in a birefringent optical fiber," Phys. Rev. Lett. 78, 3290-3293 (1997).

28. N. N. Akhmediev and J. M. Soto-Crespo, "Dynamics of solitonlike pulse propagation in birefringent optical fibers," Phys. Rev. E 49, 5742-5754 (1994)

29. S. T. Cundiff, B. C. Collings, N. N. Akhmediev, J. M. SotoCrespo, and W. H. Knox, "Polarization-locked vector solitons in a fiber laser," presented at Nonlinear Optics '98: Materials, Fundamentals and Applications Topical Meeting, Princeville, Kauai, Hawaii, August 10-14, 1998, paper TuB3.

30. S. T. Cundiff, B. C. Collings, N. N. Akhmediev, J. M. Soto-
Crespo, K. Bergman, and W. H. Knox, "Observation of polarization-locked vector solitons in optical fiber," Phys. Rev. Lett. 82, 3988-3991 (1999).

31. B. C. Collings, S. T. Cundiff, N. N. Akhmediev, J. M. SotoCrespo, K. Bergman, and W. H. Knox, "Polarization-locked temporal vector solitons in a fiber laser: experiment," $\mathrm{J}$. Opt. Soc. Am. B 17, 354-365 (2000).

32. M. Hofer, M. H. Ober, R. Hofer, G. A. Reider, K. Sugden, I. Bennion, M. E. Fermann, G. Sucha, D. Harter, C. A. C. Mendonca, and T. H. Chiu, "Monolithic polarization insensitive passively mode-locked fiber laser," in Optical Fiber Communication Conference, Vol. 2 of 1996 OSA Technical Digest Series (Optical Society of America, Washington, D.C., 1996), pp. 4-5.

33. C. Paré and P. A. Bélanger, "Optical solitary waves in the presence of a Lorentzian gain line: limitations of the Ginzburg-Landau model," Opt. Commun. 145, 385-392 (1998).

34. L. W. Liou and G. P. Agarwal, "Solitons in fiber amplifiers beyond the parabolic-gain and rate-equation approximations," Opt. Commun. 124, 500-504 (1996).

35. N. N. Akhmediev, A. Ankiewicz, M. J. Lederer, and B. Luther-Davies, "Ultrashort pulses generated by modelocked lasers with either a slow or a fast saturable-absorber response," Opt. Lett. 23, 280-282 (1998).

36. J. M. Soto-Crespo, N. N. Akhmediev, and V. V. Afanasjev, "Stability of the pulselike solutions of the quintic complex Ginzburg-Landau equation,” J. Opt. Soc. Am. B 13, 14391449 (1996).

37. C. Hönninger, R. Paschota, F. Morier-Genoud, M. Moser, and U. Keller, " $Q$-switching stability limits of continuouswave passive mode locking," J. Opt. Soc. Am. B 16, 46-56 (1999).

38. E. Desurvire, Erbium-Doped Fiber Amplifiers (Wiley, New York, 1994).

39. G. P. Agrawal, Nonlinear Fiber Optics, 2nd Ed. (Academic, San Diego, Calif., 1995). 